\title{
ASPECTOS DEFLACIONARIOS DE LA DEVALUACIÓN DEL PESO MEXICANO DE 1976 *
}

\author{
José CóRdoba y GuILleRmo OrTIZ \\ Mayo, 1979
}

ESTE DOCUMENTo forma parte de un estudio más amplio de las causas y consecuencias de la devaluación que en 1976 sufrió el peso mexicano. Aquí trataremos específicamente los efectos deflacionarios que produjo la devaluación en la demanda global, como consecuencia de la reducción de la inversión privada, hecho que probablemente constituye la característica más notable de la experiencia mexicana.

El documento se divide en cuatro secciones. En primer lugar, se presentará un resumen histórico de los eventos que llevaron al abandono de la paridad fija del peso en relación con el dólar, que prevaleció ininterrumpidamente durante 22 años. En la segunda sección, se hará una breve revisión de la literatura sobre devaluación que no proporcionó un modelo satisfactorio que pudiera ser aplicado al caso de México; luego se presenta un modelo de los efectos de la devaluación sobre la decisión empresaria respecto de las inversiones. En la tercera sección se incluyen las estimaciones empíricas del modelo desarrollado en la sección anterior y, finalmente, en la última parte, se presenta un resumen de las conclusiones y algunas perspectivas para realizar investigaciones futuras.

* Nota: Este documento se presentó en la Reunión Anual de la Eastern Economic Association que se celebró del 10 al 12 de mayo de 1979 en Boston, Mass. Traducción del inglés realizada por la Oficina de Traducciones del Banco de México, S. A.

Las ideas contenidas en el presente ensayo son responsabilidad exclusiva de los autores y no reflejan la posición del Banco de México, S. A.

* Expresamos nuestro agradecimiento a Patricia Abreu, Justino de la Cruz y Decio de María por la valiosa ayuda que nos brindaron en las investigaciones, y a Ernesto Pérez Rubio y Ciro Vergara por su colaboración en el arreglo de los archivos de computación, base de los datos. También agradecemos a Jaime Corredor el habernos facilitado el acceso a los datos compilados por la Oficina de Asesores de la Presidencia de la República. Asimismo agradecemos el apoyo financiero que nos otorgaron el Banco de México, S. A. y El Colegio de México.

** Universidad de Pennsylvania y El Colegio de México, y Banco de México, respectivamente. 


\section{La devaluación del peso en México en 1976: Un repaso}

1.1. Antecedentes históricos: Desarrollo estabilizador y la administra. ción Echeverría

La era del "desarrollo estabilizador" que se registró en los últimos años de la década de los cincuenta y la década de los sesenta, probablemente es el período de la historia económica de México ${ }^{1}$ que más se ha estudiado. Por lo tanto, deseamos presentar aquí únicamente una revisión breve de los hechos económicos de ese período y un resumen de las principales políticas desarrolladas por el gobierno. Después examinaremos los años de la administración Echeverría, el fin del desarrollo estabilizador y la secuencia de eventos que llevaron a la devaluación de $1976 .{ }^{2}$

Usando como base los dos indicadores más comúnmente empleados para medir el comportamiento económico - la tasa de crecimiento de la producción real y de los precios-, la evolución de la actividad económica durante el período de desarrollo estabilizador fue muy distinta de la experimentada durante los años 40 y parte de los 50 . La tasa media de crecimiento de la producción real de 1958 a 1970 fue de $6.67 \%$, en tanto que la del deflactor del PNB fue de $3.8 \%$. En contraste, durante el periodo anterior (de 1940 a 1957), el crecimiento de la producción y los precios fue de $6.0 \%$ y $10.2 \%$, respectivamente.

La estructura de la producción también varió. La participación del sector agrícola en el PNB se redujo de $10.8 \%$ durante el periodo inflacionario, a $9.1 \%$ en los años de desarrollo estabilizador, en tanto que la participación del sector industrial aumentó de $25.9 \%$ a $30.9 \%$. En particular, el sector manufacturero crecí́ a una tasa promedio de $8.6 \%$ en el período de estabilización, aumentando su participación en el PNB de $17.1 \%$

\section{Cuadro 1}

LA ESTRUCTURA de LA PRODUCCIóN: 1945-1970 (participación media)

\begin{tabular}{lcc}
\hline & $1945-1957$ & $1958-1970$ \\
\hline Agricultura & 18.08 & 14.57 \\
Industria. & 26.53 & 30.84 \\
Servicios & 55.39 & 54.59 \\
& 100.00 & 100.00
\end{tabular}

Fuente: Banco de México, S. A., Informes Anuales.

1 En Banco de México (1979) puede encontrarse extensa bibliografía.

2 Solís (1979) da una explicación muy amplia de la administración Echeverría. 
en 1950 a $22.8 \%$ en 1970 . Por otra parte, el tamaño del sector externo como proporción de la demanda global se redujo de un promedio de $19.9 \%$ durante 1940-1957 a $13.7 \%$-también en promedio- entre 1958 y 1970.

La evolución de la estructura productiva tuvo un desarrollo acorde con las políticas económicas que se establecieron. Se aplicaron incentivos fiscales, medidas proteccionistas y la asignación del gasto público a fin de promover una rápida industrialización durante los años sesenta.

Según Antonio Ortiz Mena, Secretario de Hacienda de 1958 a 1970, "los ingresos fiscales se dedicaron al objetivo de aumentar las existencias de capital por trabajador empleado, y a absorber, en las actividades más productivas, el aumento en la fuerza laboral"', lo que significó que la carga fiscal se mantuviera a niveles extremadamente bajos incluso en relación con los que registraban los países en desarrollo. ${ }^{4}$

Mediante medidas proteccionistas (tarifas y derechos aduaneros) se puso en práctica la política de sustitución de importaciones, al igual que en casi todos los países que siguieron este esquema de desarrollo. Aunque no se elevó en forma significativa el nivel de protección efectiva entre 1960 y 1970 , la proporción del total de las importaciones que requerían permiso aumentó de $34.3 \%$ a $68.3 \%$ durante este período. Dichas políticas se convirtieron (al igual que en muchos otros países en desarrollo) en un patrón de crecimiento orientado hacia adentro, y generaron una menor participación del sector externo en la demanda global y una estructura de costos que operó en contra de las exportaciones tradicionales. ${ }^{5}$

Además, de acuerdo con la dirección de la política fiscal y comercial, el gasto público se orientó hacia la promoción del crecimiento industrial. La participación de la agricultura en las inversiones públicas se redujo de un $20 \%$ estimado entre 1947 y 1957 a un poco más del $10 \%$ en la segunda mitad de los sesentas; en consecuencia, el valor de los productos agrícolas como proporción de la producción total también se redujo de $18.1 \%$ a $13.1 \%$ en el mismo período. Asimismo, se dio mayor importancia a la inversión pública en infraestructura de fomento industrial, en comunicaciones y energéticos durante el período de desarrollo estabilizador. El gobierno se convirtió en proveedor de servicios y materias primas a precios subsidiados.

Como se mencionó anteriormente, aunque la participación del ingreso fiscal premaneció prácticamente inalterada durante los sesentas, el gasto del sector público se incrementó a un ritmo mucho más acelerado. El déficit provocado debió financiarse, en su mayor parte, con endeudamiento interno $\mathrm{y}$ externo.

3 Ortiz Mena (1969).

4 Durante los sesentas, la participación del ingreso federal en el PNB fue aproximadamente del $8 \%$, en tanto que el ahorro corriente del gobierno se redujo de $1.7 \%$ del PNB en 1960 a $-1.9 \%$ en 1970.

5 Ver los estudios de CEPAL-NAFINSA (1971) y G. Bueno (1971). 
El elevado ritmo de crecimiento del mercado de capital internacional en los sesentas facilitó el acceso de México a fondos extranjeros; los créditos provenientes del exterior aumentaron en un promedio de $15.2 \%$ entre 1960 y 1970 , incrementando su participación en el ingreso de $6.7 \%$ a $9.8 \%$. El endeudamiento interno también se hizo posible debido al crecimiento espectacular que registró el mercado financiero doméstico. Como se muestra en el cuadro 2, el total de las obligaciones de las instituciones financieras (excluyendo al Banco de México) aumentó a una tasa promedio de $12.9 \%$ entre 1955 y 1970 , y se incrementó, como proporción del PNB, de $20.4 \%$ a $45.6 \%$ en esos años. La tasa de crecimiento del crédito real, durante el período $1960-1970$, fue de $12.3 \%$, es decir el doble del crecimiento de la producción.

Cuadro 2

INDICADORES FINANCIEROS: 1945-1970

(tasas del crecimiento medio real)

\begin{tabular}{lcc}
\hline & $1945-1957$ & $195.8-1970$ \\
\hline Obligaciones monetarias & 1.65 & 3.03 \\
Obligaciones. no monetarias & 7.36 & 14.27 \\
Total de creditos otorgados & 7.08 & 13.06
\end{tabular}

Fuente: Banco de México, S. A. Informes Anuales.

El aumento de la intermediación financiera es el factor clave en la explicación de la estabilidad de los sesentas. ${ }^{6}$ El gobierno pudo captar ahorros privados voluntarios para financiar sus déficits, conservando la tasa de crecimiento del medio circulante a un nivel del $10.6 \%$ durante esa década. Como consecuencia, el ajuste a la demanda excedente en el mercado de bienes, producto de los gastos gubernamentales, se canalizó principalmente a través de la balanza de pagos y no mediante el aumento de los precios internos.

Por lo tanto, el sistema fue congruente y estuvo totalmente determinado por los objetivos (tasa de crecimiento de la producción, estabilidad de precios y tipo de cambio fijo) e instrumentos (encaje legal, gasto público y créditos del exterior). Evidentemente, la restricción consistía en mantener una coherencia entre el comportamiento de los instrumentos y los objetivos: los límites del gasto público (y por lo tanto del crecimiento de la producción) estaban dados por las posibilidades de endeudamiento externo e interno, que aseguraban el logro de los otros dos objetivos.

6 También hay amplia literatura respecto de este punto. Ver por ejemplo, Brothers y Solís (1966).

7 Véase Blejer (1977). 
Cuadro 3

RESUMEN DE LA BALANZA DE PAgOS: 1965-1976

\begin{tabular}{|c|c|c|c|c|}
\hline & $\begin{array}{l}\text { Cuenta } \\
\text { Corriente }\end{array}$ & $\begin{array}{l}\text { Errores y } \\
\text { Omisiones }\end{array}$ & $\begin{array}{c}\text { Capital a } \\
\text { Largo Plazo }\end{array}$ & $\begin{array}{l}\text { Cambios } \\
\text { Ias Reservas }\end{array}$ \\
\hline 2965 & $-\quad 357$ & 252 & 94 & -21 \\
\hline 1966 & $-\quad 391$ & 174 & 224 & 6 \\
\hline 1967 & $-\quad 506$ & 200 & 246 & 40 \\
\hline 1968 & 632 & 302. & 379 & 49 \\
\hline 1969 & -473 & 172 & 693 & 48 \\
\hline \pm 970 & -946 & 499 & 504 & 102 \\
\hline $1965-1970$ & -3315 & 1255 & 2240 & 224 \\
\hline 1971 & -726 & 218 & 669 & 200 \\
\hline 1972 & $-\quad 762$ & 234 & 754 & 265 \\
\hline 1973 & -1.175 & 378 & 1676 & 122 \\
\hline 1974 & -2558 & $-\quad 136$ & 2731 & 37 \\
\hline 1975 & -3769 & $-\quad 406$ & 4340 & 165 \\
\hline 1976 & -3044 & -2596 & 4595 & -333 \\
\hline
\end{tabular}

Fuente: Banco de México, Informes Anuales.

A fines de la década del 60 , se observaron indicios de que la balanza de pagos se había vuelto más sensible al gasto global.

Como se desprende del cuadro 3, en 1970, el déficit en cuenta corriente de la balanza de pagos fue casi el doble del que se registró el año anterior. La nueva administración, preocupada por la magnitud del déficit, decidió una reducción considerable del gasto público para el presupuesto de 1971. Sin embargo, el gasto real fue aún más bajo que el presupuestado y el déficit fiscal -como porcentaje del PNB-: se redujo de $1.5 \%$ en 1970 a $1.05 \%$ en 1971 . Consecuentemente, gran parte de los ahorros financieros reservados al sector público permanecieron sin aplicación, y aunque el aumento del crédito real del sector financiero otorgado a los prestatarios privados permaneció a un nivel normal, los bancos acumularon reservas por 2700 millones de pesos. ${ }^{8}$ Esto determinó una baja repentina de la actividad económica en 1971. La producción real creció apenas un 3.4\% (la cifra más baja de los últimos doce años) mientras que los pronósticos previos habían anticipado una tasa de crecimiento aproximada de $5 \%$.

Retrospectivamente, podemos argüir que 1972 marcó el fin del período de desarrollo estabilizador. Aunque se alcanzó el objetivo de reducir el crecimiento del déficit en cuenta corriente (ver cuadro 3), el

8 Solis (1979). 
costo resultó demasiado alto. La severa contracción de la actividad eco nómica que se experimentó en 1971 causó gran preocupación entre los funcionarios gubernamentales, especialmente debido a que las políticas restrictivas aplicadas contradecían el discurso reformista de la nueva administración.

"Cuando se conoció el deterioro del crecimiento del PNB en 1971, y se hizo pública la existencia de reservas bancarias excedentes, invertidas en depósitos rentables en el Banco Central, en muchos ámbitos se hizo patente el sentimiento de la urgencia de restaurar la tasa tradicional de crecimiento" (Solís: 1979, p. 87). Para ello, se incrementó rápidamente el gasto público y se estimuló el gasto privado. El déficit del sector público, como proporción del PNB, ascendió a más del triple entre 1971 y 1972 y se utilizó la política monetaria para promover la inversión privada: la tasa de depósito sobre reservas excedentes se redujo a principios do 1972 y el encaje legal también se redujo en el mes de mayo. Sin embargo, parecería que se implantaron políticas expansionistas varios meses después de que la tasa de crecimiento de la producción industrial (el único indicador disponible de la utilización de la capacidad) había superado su tendencia de largo plazo. Como resultado de esto, tanto la producción como los precios mostraron una fuerte reacción, con la diferencia que estos últimos lo hicieron con retraso: la tasa de crecimiento del PNB en 1972 fue de más del doble de la registrada el año anterior, en tanto que el incremento de los índices de precios al consumidor y al por mayor mostraron, respectivamente, tasas moderadas de $4.9 \%$ y $2.8 \%$. Sin embargo, a mediados de 1972, se observó claramente que los precios comenzaron a elevarse.

En enero de 1973, el incremento del índice de precios al por mayor fue de $7.3 \%$, y en diciembre del mismo año, había aumentado a $25.5 \%$, superando en mucho la tasa observada en los Estados Unidos. Debido al gran volumen de comercio que se registra con los Estados Unidos, la aceleración de la inflación en México y el agudo incremento del déficit de la balanza de pagos que se observó en 1973 (como consecuencia del rápido crecimiento en el ingreso nominal) debilitaron seriamente la posición del peso; los capitales a corto plazo fueron retirándose del país.

Por otra parte, como resultado de las condiciones desfavorables en la economía mundial, la demanda externa de productos mexicanos también se redujo, en tanto que el fuerte incremento de los precios del petróleo, junto con dos años especialmente adversos para la agricultura, tuvieron un efecto negativo sobre las importaciones. Como consecuencia, el déficit comercial se elevó de 1700 millones de dólares en 1973 a 3100 millones en 1974 y a 3600 en 1976. Naturalmente, esto sólo fue posible realizarlo con el incremento de la deuda externa. El total de la deuda externa del sector público aumentó de 3300 millones de dólares en 1970 a 16000 millones a fines de 1976.

Los efectos, tanto de la inflación como de la creciente incertidumbre 
en el aspecto cambiario, se dejaron sentir rápidamente en el sector financiero. La relación ahorro financiero/PNB, que en 1972 alcanzó su nivel más alto, comenzó a declinar en 1973 -el primer año de alta inflación y tasas negativas de interés real- y en 1974. La estructura de las obligaciones (cuadro 4) también se modificó. La tasa de crecimiento del ahorro canalizado a través de los diversos instrumentos financieros sufrió una severa reducción; la tasa de crecimiento media, en términos reales, de estos valores sólo fue 1/6 de la tasa observada entre 1965 y 1970.

\section{Cuadro 4}

AHORROS FINANCIEROS: 1971-1976

\begin{tabular}{|c|c|c|c|c|c|c|c|}
\hline \multirow[b]{2}{*}{ Aros } & \multicolumn{4}{|c|}{ Tasa de crecimiento real } & \multicolumn{2}{|c|}{ Proporciones } & \multirow[b]{2}{*}{$\begin{array}{l}\text { Obligaciones } \\
\text { denominadas } \\
\text { en dólares }\end{array}$} \\
\hline & $\begin{array}{l}\text { Depositos } \\
\text { a la } \\
\text { vista }\end{array}$ & $\begin{array}{l}\text { Depósitos } \\
\text { de } \\
\text { Ahorros }\end{array}$ & Bonos & Certificados & $\begin{array}{c}\text { No } \\
\text { Monetarias }\end{array}$ & Monetarias & \\
\hline 1971 & 3.39 & 9.70 & 20.99 & 12.75 & 60.4 & .22 .5 & 17.1 \\
\hline 1972 & 14.32 & 11.13 & 15.33 & 21.95 & 80.7 & 23.6 & 15.7 \\
\hline 1973 & 7.86 & 8.60 & -2.70 & -2.47 & 56.1 & 24.9 & 19.0 \\
\hline 1974 & -1.10 & -2.88 & -24.65 & -8.86 & 53.8 & 25.4 & 20.8 \\
\hline 1975 & 2.19 & 1.26 & -1.60 & 10.70 & 53.3 & 24.5 & 22.2 \\
\hline 1976 & -4.44 & -11.68 & -34.83 & -18.78 & 37.7 & 22.6 & 39.7 \\
\hline
\end{tabular}

Fuente: Banco de México, Informes Anuales.

La reducción del ahorro financiero tuvo efectos adversos notables en la economía como un todo. Primero, porque el gasto público no podía financiarse adecuadamente con fondos no inflacionarios como se hizo en el pasado, especialmente debido a que su proporción del PNB era mucho mayor que en los años anteriores; en segundo lugar, porque se redujo el financiamiento privado; en tercero -y quizá el más importante- porque al no respetarse la restricción presupuestaria, el gobierno comenzó a perder dos de sus instrumentos de control monetario: la administración del encaje legal y el endeudamiento externo. ${ }^{9}$ Las modificaciones del encaje legal sólo son eficaces en la medida que afecten el crédito otorgado al sector privado. Si el déficit gubernamental es de suficiente magnitud como para forzar un desplazamiento importante del endeudamiento privado, el encaje legal dejará de ser un instrumento de política monetaria, dado que estará fijado sólo por el tamaño del déficit. Si, por otra parte, el endeudamiento interno no puede sustituirse con endeudamiento externo debido a la gran proporción de la deuda externa acumulada, este otro instrumento de política también podrá perderse. Los intentos para hacer más

9 Para un comentario más amplio de este punto, véase Ortiz y Solís (1978). 
eficiente la economía y los reveses experimentados entre 1973 y 1975 aparentemente confirman este punto.

Aunque a mediados de 1973 se anunciaron estrictas medidas antiinflacionarias, el déficit del sector público continuó aumentando. De un nivel de $2.2 \%$ en 1971, aumentó a $5.1 \%$ en 1973 y en 1975 alcanzó $9.0 \%$. El repunte de la inflación tuvo un típico efecto de incremento del déficit al aumentar los gastos corrientes que fueron difíciles de recortar a pesar de las intenciones expresadas. En resumen, la secuencia de eventos que llevaron a la devaluación puede describirse como sigue: después de la recesión de 1971, el gobierno siguió una política simultánea de gasto público y expansión crediticia. El déficit gubernamental se monetizó rápidamente (la tasa de crecimiento de $M_{1}$ creció a más del doble), y conforme se agotaba la capacidad excedente, los precios comenzaron a subir con rapidez. Las tasas de interés negativas desalentaron el crecimiento de los ahorros financieros y se redujo la fuente de fondo más importante tanto para el sector público como para el privado. El impacto de los aumentos en la demanda global y la inflación sobre la balanza comercial, forzaron al gobierno a incrementar el endeudamiento externo para evitar una disminución de las reservas; pero todo esto se añadió al temor creciente de una devaluación y se aceleró el éxodo de capitales.

\subsection{Devaluación: Las consecuencias}

El 31 de agosto de 1976, en vista del creciente éxodo de capitales, el Secretario de Hacienda anunció que se abandonaba la paridad fija (de 12.50 pesos por dólar) que estaba vigente desde 1954. Se dejaría que el peso flotara en relación al dólar a fin de que el mercado determinara su "valor de equilibrio". El tipo de cambio se elevó inmediatamente a 20-21 pesos por dólar y el Banco Central comenzó a manejar una reducción gradual de la relación peso-dólar decretando una paridad temporal de 19.7019.90 pesos por dólar. Entre tanto, se habían iniciado las negociaciones con el FMI; a fines de octubre, se aprobó un crédito inicial de $837 \mathrm{mi}$ llones de DEG's. El Departamento del Tesoro de los Estados Unidos y el Sistema de la Reserva Federal también accedieron a proporcionar un crédito "swap" de 600 millones. A pesar de este apoyo, las incesantes fugas de capital forzaron al Banco de México a liberar de nuevo el peso y esta vez el tipo de cambio alcanzó nuevos niveles (24.00-24.50). En esta ocasión (la tercera semana de noviembre), el Banco Central dictó una suspensión indefinida de las operaciones cambiarias, permitiendo que las casas de cambio instauraran un mercado paralelo. Los bancos privados reiniciaron las operaciones cambiarias algunas semanas después que hubo tomado posesión la nueva administración y que se disipó la fiebre especulativa. Desde entonces, el peso ha registrado algunos repuntes respecto al dólar y desde septiembre de 1977 se ha mantenido prácticamente fijo en un nivel promedio de 22.75 pesos por dólar. 
La magnitud de la devaluación (45\%) fue muy superior a la anticipada en general..$^{10}$ No es de sorprender que la devaluación haya tenido importantes efectos en muchos aspectos de la actividad económica. ${ }^{11}$

Como era de esperarse, la balanza de pagos mostró una notable mejoría después de la devaluación. El déficit en cuenta corriente en 1977 fue de 1700 millones de dólares, $41.5 \%$ más bajo que el registrado el año anterior; también, si se excluyen los pagos en concepto de deudas y los intereses, la cuenta corriente mostró un superávit de 406 millones. Sin embargo, la mejora fue más la consecuencia de una reducción de las importaciones que del aumento de las exportaciones, reflejo esto de la contracción de la actividad económica: la caída de las importaciones de bienes de capital representó el $98 \%$ de la reducción total de las importaciones (542 millones) respecto de 1976. Si se excluyen las exportaciones de petróleo, por otra parte, las exportaciones totales aumentaron únicamente en 418 millones (15\%).

Como se muestra en la gráfica 1, la contracción de la actividad económica fue muy severa. La producción real aumentó únicamente $2.8 \%$ en 1977 (índice menor, inclusive, que el del crecimiento de la población). La actividad de la producción industrial también se contrajo durante el primer semestre y comenzó a recuperarse lentamente en el tercer trimestre, mostrando un aumento del $3 \%$ a fin de año.

Es evidente que prácticamente todos los componentes de la demanda global se vieron afectados por la devaluación. Sin embargo, la caída de la inversión fue particularmente fuerte -es por ello que nuestra preocupación principal en este trabajo es el comportamiento de la inversión después de la devaluación. La producción de bienes de inversión se redujo $6.6 \%$ en 1977 respecto del año anterior, y, como se indicó anteriormente, las importaciones de bienes de capital también se redujeron en un $14 \%$. El comportamiento de ambas variables puede verse en la gráfica dos.

De acuerdo con estimaciones de las cuentas nacionales correspondientes a $1977,,^{12}$ se redujo la inversión bruta total en un $7.6 \%$ en términos reales, en tanto que el consumo privado permaneció más o menos estable. Aunque por el momento no se cuenta con información específica respecto a la distribución del gasto de consumo y de inversión, el impacto

10 Como, por ejemplo, se reflejaba en la tasa de descuento futura del peso, en Chicago y Nueva York.

11 Aún se debate la cuestión respecto de si la devaluación era "inevitable" en ese momento, o si pudo haberse pospuesto algunos meses mientras se calmaba la situación. Por su parte, Solís (1979) sostiene que la devaluación fue demorada mucho tiempo y que Echeverría pudo haber devaluado al comienzo de su administración, aprovechando que el valor del dólar había caído respecto al oro en 1971. Otro aspecto del debate, se relaciona con la división de responsabilidades respecto de los hechos que llevaron a la devaluación como una política de gobierno, la inestabilidad de la situación internacional y el legado del período de desarrollo estabilizador. Ver Reynolds (1977).

12 Banco de México (1978). 
Gráfica 1

Crecimiento de las manufacturas; tasas anUales, promedios móviles

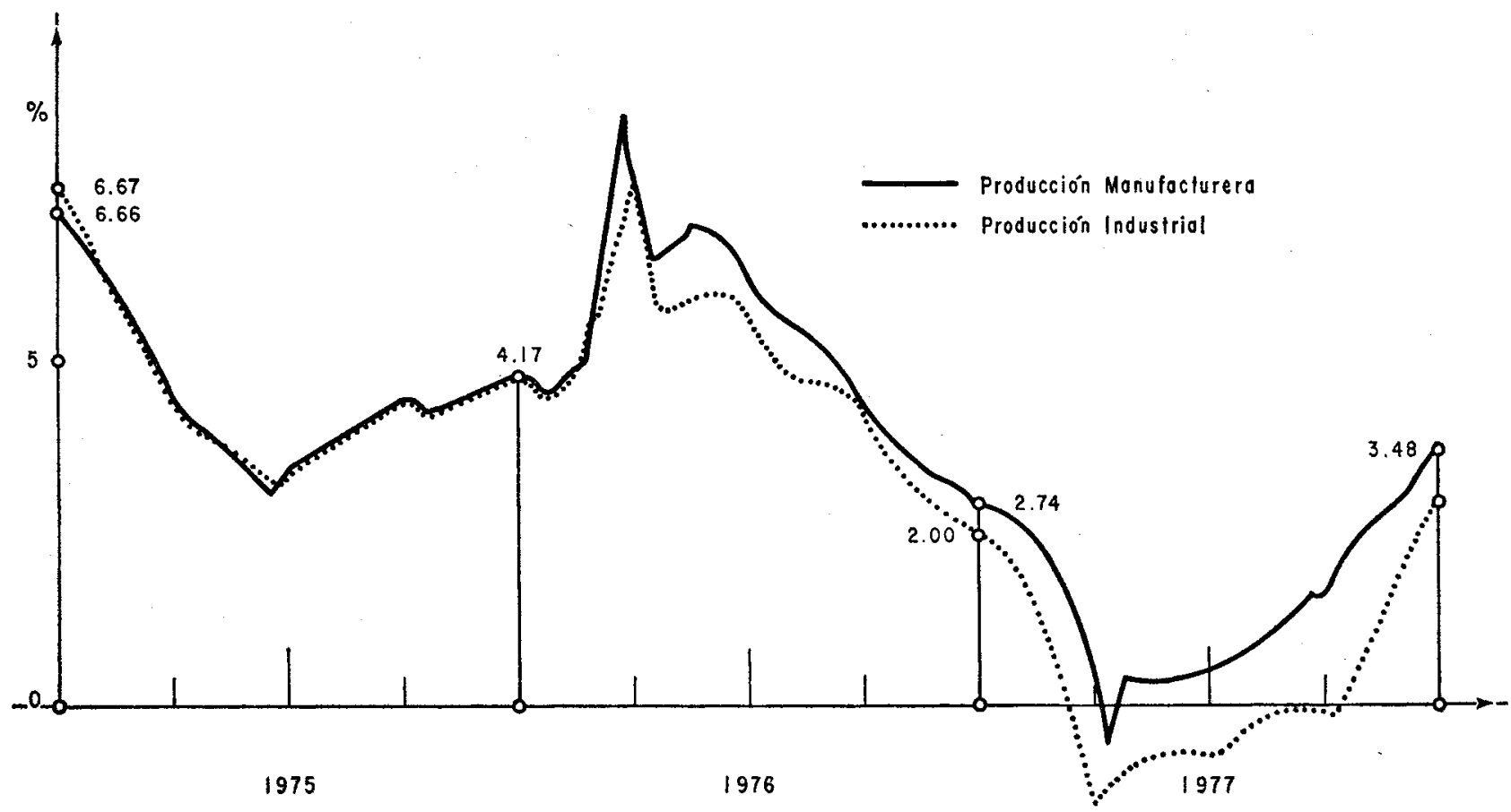

Fuente: Zepeda (1979) 
de la recesión puede apreciarse observando los datos de producción (promedios mensuales en términos reales 1977/1976): los bienes de consumo duradero se redujeron en $3.3 \%$; los bienes no duraderos aumentaron $5.7 \%$; los bienes de producción se incrementaron en $3.0 \%$ y de éstos, los bienes de inversión se redujeron en $6.6 \%$ (según se indicó anteriormente) y las materias primas aumentaron en $4.7 \%$.

\section{Gráfica 2}

IMPORTACIONES Y PRODUCCIÓN DE BIENES DE INVERSIÓN: NÚMEROS INDICES

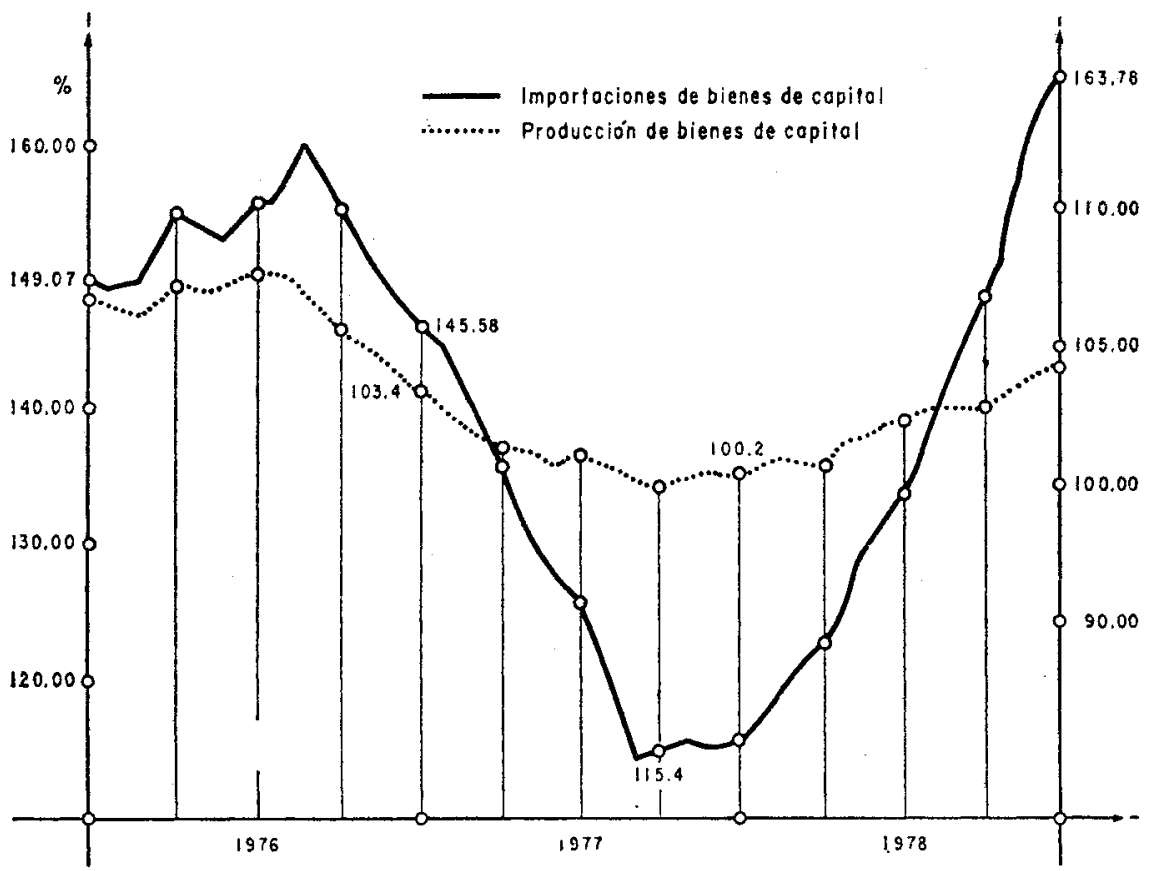

Fuente: Zepeda (1979).

El impacto sobre los precios también fue muy fuerte - como era de esperarse después de una devaluación de esa magnitud. El índice de precios mayoristas, que había estado incrementándose a una tasa anual del $14 \%$ desde enero hasta agosto de 1976, sólo en septiembre aumentó $6 \%, 8.3 \%$ en octubre, $8.2 \%$ en noviembre y $5 \%$ en diciembre. El aumento del índice de precios al consumidor lógicamente fue menor: el incremento acumulado en los seis meses que siguieron a la devaluación fue 
sólo un poco superior al $20 \% .^{13}$ También se autorizó un aumento de emergencia de $23 \%$ en los sueldos, inmediatamente después de la devaluación, a fin de prevenir un hundimiento del salario real.

La incertidumbre cambiaria que prevaleció durante el primer semestre de 1976 y que originó el éxodo masivo de capitales, como se mencionó anteriormente, no se redujo después de la devaluación; las salidas continuaron durante los últimos meses de 1976 y el proceso de sustitución de moneda (dólares por pesos) o "dolarización" de la economía, registró un ritmo más acelerado. Las obligaciones totales no monetarias denominadas en moneda nacional, en manos de las instituciones financieras privadas, se redujeron en un 10\% (21 millones de pesos) de septiembre a diciembre, en tanto que las obligaciones en dólares aumentaron en un $38 \%$ (18 millones de pesos). Durante los primeros meses de 1977, se frenó el éxodo de capitales, pero el proceso de dolarización continuó según se muestra en el cuadro 5.

\section{Cuadro 5}

FLU JOS FINANCIEROS: 1975-1977

(miles de millones de pesos)

\begin{tabular}{|c|c|c|c|c|}
\hline & $\begin{array}{l}\text { Dic.1976/ } \\
\text { Dic.1975 }\end{array}$ & $\begin{array}{l}\text { Abr. } 1977 / \\
\text { Dic. } 1976\end{array}$ & $\begin{array}{l}\text { Dic. } 1977 / \\
\text { May.1977 }\end{array}$ & $\begin{array}{l}\text { Dic. } 1977 / \\
\text { Dic. } 1976\end{array}$ \\
\hline $\begin{array}{l}\text { Cambio total"en las obli- } \\
\text { gaciones del sector fi- } \\
\text { nanciero. }\end{array}$ & 114.8 & 25.6 & 137.6 & 163.2 \\
\hline $\begin{array}{l}\text { Oblizaciones contratadas } \\
\text { en pesos }\end{array}$ & 37.2 & 9.0 & 100.5 & 109.5 \\
\hline Depositos a la Vista & 36.5 & -10.0 & 50.6 & 40.8 \\
\hline $\begin{array}{l}\text { No Konetarios (a corto } \\
\text { plazo) }\end{array}$ & -15.4 & 22.3 & -15.8 & 6.5 \\
\hline $\begin{array}{l}\text { No Monetanios (a Largo } \\
\text { plazo) }\end{array}$ & 15.1 & -3.3 & 65.7 & 62.4 \\
\hline $\begin{array}{l}\text { Oblizaciones contratadas } \\
\text { en dólares } \\
\text { (Ajustado por movimientos } \\
\text { en el tipo de cambio) }\end{array}$ & 77.6 & 16.6 & 37.1 & 53.7 \\
\hline
\end{tabular}

Fuente: Banco de México, Informe Anual 1977.

El incremento de las tasas de interés domésticas de mayo de 1977, junto con la reducción de la tasa de crecimiento de los precios que se re-

13 Puesto que el índice de precios al mayoreo contiene mayor volumen de bienes negociables, puede esperarse que su movimiento sea más rápido que el del indice de precios al consumidor, cuando se altera el tipo de cambio.

14 Las obligaciones no monetarias a corto plazo incluyen todas las obligaciones financieras con vencimiento a un mes. 
gistró en el segundo semestre de ese año, aumentó las tasas reales a niveles positivos por primera vez desde 1972. También, la intervención más activa del Banco de México en el mercado cambiario produjo menores fluctuaciones del tipo de cambio. Estas medidas evitaron que los especuladores tomaran posiciones en dólares, y lus ahorros financieros en moneda nacional se reconstituyeron gradualmente. La situación crediticia también reflejó la "dolarización" de la economía: en 1975 sólo $5 \%$ del total de los créditos otorgados por las instituciones financieras privadas se entregó en dólares; las cifras correspondientes a 1976 y 1977 son de $22 \%$ y $25 \%$ respectivamente. Debido a la restricción del crédito durante los meses que siguieron a la devaluación, los posibles prestatarios se vieron obligados a elegir entre crédito limitado y caro en moneda nacional, o aumentar su carga en dólares con el consiguiente riesgo cambiario.

La disminución de los ahorros financieros en moneda nacional, naturalmente obligó a las instituciones financieras a reducir el flujo de crédito otorgado en pesos al sector privado. Entre septiembre y diciembre de 1976, el crédito real adicional otorgado al sector privado en moneda nacional, en términos nominales, aumentó menos de la mitad del promedio registrado durante los dos años anteriores, y mostró una importante reducción en términos reales. Esta tendencia continuó durante los primeros meses de 1977. Por otra parte, después del acuerdo con el FMI, el crecimiento del déficit del sector público en términos reales, también se redujo considerablemente. En consecuencia, no es difícil apreciar los serios problemas financieros que afrontaron las empresas después de la devaluación (y especialmente las que tenían obligaciones no cubiertas en monedas extranjeras), y cómo fue que la caída de la inversión resultante de dichos problemas (como se comenta más adelante) se precipitó a causa de la propia devaluación.

\section{La devaluación y las decisiones de inversión}

\subsection{La teoría de la devaluación}

En esta sección, se analizará la capacidad de las teorías tradicionales para explicar las consecuencias económicas de la devaluación del peso en México en 1976. Esta sección se divide en dos partes. Primero se proporciona un resumen esquemático de las teorías tradicionalmente utilizadas para analizar los efectos de una devaluación. Después se presentarán los diversos efectos deflacionarios específicamente mencionados en la literatura y se señalará la relativa originalidad del caso mexicano.

\subsubsection{Breve revisión de la literatura sobre el problema tratado}

Los estudios que se refieren a los efectos de la devaluación de una moneda se han desarrollado tradicionalmente de acuerdo con 3 enfoques: 
el de la elasticidad, el de la absorción y el monetarista. En principio, estas tres perspectivas aparentemente tienen fuertes contrastes; sin embargo, después de un estudio minucioso, son más complementarias que contradictorias.

Por definición, el tipo de cambio es el precio relativo de dos monedas. En una economía de mercado libre (es decir en el que todos los precios relativos son totalmente elásticos), el valor de equilibrio del tipo de cambio está determinado por el agotamiento de las posibilidades pertinentes del arbitraje. Por otra parte, una devaluación es un cambio discontinuo y discrecional en el valor del tipo de cambio resultante del reconocimiento de algún tipo de "desequilibrio": supone tipos de cambio fijos e intervención del gobierno.

Desde el punto de vista institucional, se excluyen mutuamente un mercado cambiario libre y un sistema de paridad fija. Desde el punto de vista teórico, sin embargo, la determinación del valor de equilibrio de paridad en un sistema de mercado libre y la determinación de los efectos económicos posteriores a la devaluación en un sistema de paridad fija son dos problemas profundamente relacionados, simétricos en un sentido casi dual: los mismos canales que determinan el tipo de cambio de equilibrio son precisamente aquellos a través de los cuales se dejarán sentir los efectos de una devaluación. Una vez reconocida la simetría, la relación entre los tres enfoques antes mencionados parece menos antagónica. En tanto que el enfoque de elasticidad y el de la absorción corresponden a una teoría parcial de la devaluación sin una teoría explícita de la determinación del tipo de cambio de equilibrio, el enfoque monetarista consiste básicamente en una teoría del equilibrio de los mercados cambiarios, con una teoría implícita de la devaluación. Las discrepancias entre estas tres perspectivas se deben, en mayor grado, a las diferencias de cobertura y énfasis, que a desacuerdos sustanciales.

En gran medida, las controversias de la literatura relativa a la determinación y ajuste del tipo de cambio, son parecidas a las que tratan la determinación y ajuste de la tasa de interés. No es de sorprender que las tasas de interés y los tipos de cambio se encuentren entre los precios únicos más importantes a nivel macroeconómico, sino los más importantes: expresado en forma sencilla, la tasa de interés es el precio del futuro (respecto del presente) en tanto que el tipo de cambio es el precio de bienes y activos extranjeros respecto de la economía nacional. Desde el punto de vista práctico, esta similitud incluye una frecuente intervención estatal tanto en el mercado financiero nacional como en el mercado cambiario. Desde el punto de vista teórico, la similitud radica en el hecho de que, tanto el tipo de cambio como la tasa de interés, expresan precios relativos respecto a un numerario con valor variable. En un momento determinado, los precios nominales de los bienes y servicios nacionales se expresan en términos de un numerario que es único y uniforme (el "dólar", "el peso", etc.)) : la relación de dos precios nominales queda, por lo 
tanto, determinada por factores reales, excluyendo las variables monetarias. Sin embargo, esto no se aplica cuando se comparan los precios domésticos corrientes con precios ya sea a futuro o extranjeros. En el primer caso, la unidad de cuenta no es uniforme sino variable, puesto que la evolución de su valor (en el sentido de su poder adquisitivo) queda determinada por factores monetarios (esencialmente, la tasa de inflación). En el segundo, la unidad de cuenta no es única, sino doble, dado que dos paises diferentes generalmente utilizan dos numerarios monetarios distintos. Únicamente cuando el numerario local sea un bien real (oro, por ejemplo), se puede considerar como variable real, la relación entre los precios nominales futuros y los actuales. Sólo cuando los numerarios nacionales y extranjeros sean ambos bienes reales (idénticos, como en el sistema de patrón oro o diferentes, como en el sistema bimetálico), la relación entre los precios nominales extranjeros y los nacionales será una variable real. En cualquier otra circunstancia, los factores monetarios $y$, por lo tanto, las expectativas respecto de la política monetaria, deben ser tomadas en cuenta al determinar los precios de equilibrio relativo.

Es bien sabido ${ }^{15}$ que cuando se introducen expectativas, y a menos que se considere una perfecta previsión, difieren los conceptos del equilibrio entre existencias y flujos en los mercados de bienes. En el caso de las tasas de interés, esta divergencia corresponde a la discrepancia entre los puntos de vista de "preferencia por la liquidez" y los "fondos prestables" en los mercados crediticios. En el caso de los tipos de cambio, la divergencia corresponde, en gran parte, a la discrepancia entre el concepto del equilibrio del flujo implícito en el enfoque de elasticidades, y el concepto de equilibrio de las existencias, básico en el enfoque monetarista.

Resumiendo, el enfoque de la elasticidad enfatiza el efecto de sustitución entre productos negociados y no negociados, tanto en el consumo como en la producción, inducido por el cambio de precios relativos resultante de la devaluación. Dentro de este enfoque, puede establecerse una condición para mejorar la balanza comercial después de la devaluación, que relaciona la elasticidad de la oferta de las exportaciones, tanto a nivel nacional como internacional, y la elasticidad de la demanda de las importaciones tanto a nivel nacional como internacional; en el caso de que la oferta de exportaciones sea perfectamente elástica, esta condición se vuelve simplemente la celebrada condición de Marshall y Lerner. Sin embargo, implícita dentro de este marco analítico, está la idea de que el tipo de cambio de equilibrio lo determina el flujo de la oferta y la demanda de las exportaciones y las importaciones (esta idea está expresada con mayor claridad en el primer artículo de Joan Robinson (1937) que en artículos más recientes dentro de la tradición de la "elasticidad"). Por otra parte, es importante señalar que desde un principio se identificó el éxito de una devaluación con la condición de estabilidad del equilibrio

15 Véase Foley (1975). 
en el mercado cambiario. ${ }^{16}$ Sin embargo, el hecho de que la condición de Marshall y Lerner no sea ni la condición de estabilidad del mercado cambiario ni la condición para una devaluación favorable en una economía monetaria "genuina", fue probado por Negishi (1968) y F. Hahn (1959) y sobre todo se afianzó posteriormente la tradición monetarista.

El enfoque monetarista se concentra explícitamente en las condiciones de equilibrio de los activos en los mercados de bienes. El tipo de cambio, a corto plazo, se determina de acuerdo con el teorema de la paridad de las tasas de interés: conociéndose las tasas de interés internas y externas, la evolución esperada del tipo de cambio (medida por la prima de descuento en el mercado a futuro) debe ser igual al diferencial de las tasas de interés; por lo tanto, a corto plazo, una demanda excesiva de bienes, servicios y valores que produzca un déficit en la balanza de pagos y que refleje una oferta excedente de dinero, puede absorberse mediante ajustes, ya sea en la tasa de interés interna, o en el tipo de cambio en plaza (spot) o a futuro. Sin embargo, se considera que, a largo plazo, este grado de libertad se pierde y las expectativas se dirimen según alguna versión del teorema de la paridad del poder adquisitivo, expresando una condición de equilibrio de flujos en el mercado de bienes. ${ }^{17}$

El enfoque monetarista, de la determinación del equilibrio en el tipo de cambio, acarrea -más o menos directamente- una teoría de la devaluación (ver R. Dornbusch (1973 a) y 1973 b)).

Esta teoría enfatiza la función del mercado de activos accionarios -y más específicamente la función del valor real de los saldos en efectivoen el proceso de ajuste a un cambio en la paridad. Para una política monetaria determinada, el incremento del precio interno de los bienes negociables producido por la devaluación, determina un nivel más alto de precios internos. El menor valor real de las tenencias monetarias ejercerá un efecto-riqueza negativo sobre los gastos corrientes y originará una depresión en la demanda global interna tanto para bienes nacionales como extranjeros. La mayor demanda externa de exportaciones nacionales inducida por el cambio en el precio relativo, contribuirá además a un mejoramiento de la balanza comercial y conservará el nivel de la producción

16 Por ejemplo, véase G. Haberler (1949).

$17 \mathrm{La}$ interacción entre los mercados de activos y de bienes es mucho más compleja en el caso de la determinación de tipos de cambio que de tasas de interés. Con respecto a las tasas de interés, el precio del capital determinado en los mercados de activos, induce una oferta de equilibrio de bienes de inversión en el mercado de bienes (ver J. Tobin (1969) y D. Foley (1971)). En cuanto a los tipos de cambio, las condiciones de equilibrio en los mercados de bienes negociables tiene una influencia retroactiva - a través de su impacto en las expectativasen la determinación de los tipos de cambio en los mercados de activos. Aparentemente no existe un análisis plenamente satisfactorio del proceso de ajuste conjunto de los mercados de bienes y de activos accionarios (sin embargo, véase Dornbusch (1976) y P. Kouri (1978)). 
nacional en tanto prosigue la acumulación de activos. Al final del proceso de ajuste, los saldos reales internos se restablecerán al nivel deseado.

Al igual que el enfoque monetarista, el enfoque de absorción (iniciado con S. Alexander (1952)) enfatiza la función de una discrepancia entre gastos e ingresos -que conlleva la acumulación o dispersión de bienesen el proceso de ajuste a un cambio en la paridad. Sin embargo, no concede mucha importancia a las variaciones de los precios relativos - como lo hace, en principio aunque no siempre en la práctica, el enfoque monetarista - y no trae a primer plano la teoría implícita del equilibrio de la determinación del tipo de cambio; más específicamente, no analiza, de manera explícita, el grado en que un déficit de pagos externos se origina por un tipo de cambio en desequilibrio, o a la posición de equilibrio externo que puede resultar de una discrepancia entre las propensiones marginales externas e internas al ahorro y la productividad del capital. En retrospectiva, aparentemente, el enfoque monetarista ha integrado completamente la contribución hecha con anterioridad por la tradición de la "absorción".

\subsubsection{Los efectos deflacionarios de una devaluación}

De acuerdo con los tres enfoques tradicionales ya mencionados, no deberían registrarse efectos deflacionarios muy fuertes (en el sentido de una reducción en el nivel de la actividad económica) después de una devaluación. Aparentemente, es todo lo contrario. Si existe capacidad no utilizada en el sector de bienes negociables, el enfoque de la elasticidad predeciría una expansión en la producción nacional, inducida por una mayor demanda externa de exportaciones y una mayor demanda local de productos que puedan competir con los importados; esta predicción ha sido durante mucho tiempo la base de las llamadas "devaluaciones competitivas" y el fundamento de los gobiernos para aplicar las políticas de "reciprocidad" en tiempos de recesión. Según el enfoque de la absorción, se requiere una reducción del gasto interno para que una devaluación mejore la balanza comercial; el hecho de que tal reducción no esté automáticamente garantizada, precisamente ha llevado a prescribir medidas restrictivas monetarias y fiscales, precisamente después de proceder a una devaluación: el Fondo Monetario Internacional ha ganado cierta fama por el diseño de estos paquetes de políticas. Finalmente, es cierto que el efecto riqueza negativo subrayado por el enfoque monetarista genera la posibilidad de una reducción de la demanda global interna que apareja una reducción de la producción interna; sin embargo, esta posibilidad no ha sido estudiada a fondo y parece que en general se considera que los efectos expansivos de una mayor demanda externa de las exportaciones, compensará cualquier influencia depresiva producida por una demanda interna restringida de productos nacionales; de esta manera podría obte- 
nerse la mejora de la balanza comercial sin reducción del nivel de la actividad económica.

En la práctica, muchas devaluaciones - sobre todo en los países en desarrollo (ver R. Cooper (1971 a)) - han sido seguidas por algún tipo de recesión económica; sin embargo no siempre es claro, si es que -y por qué- el menor nivel de actividad económica puede atribuirse directamente al cambio en la paridad. Diversos estudios han ilustrado esta cuestión mediante un análisis específico de algunos efectos deflacionarios asociados con una devaluación.

R. Cooper (1971 b) ha demostrado que, si se parte de una posición inicial de déficit comercial, una devaluación puede ejercer un efecto depresivo en la actividad económica. Este argumento puede formularse de la siguiente manera: considérese la relación contable $Y=E+D$, en donde $Y$ es la producción interna, $E$ es el total de gasto interno y $D$ es la balanza comercial medida en términos de la moneda nacional. Claramente, el impacto inicial de la devaluación será deflacionario si $\Delta D$ es negativo. Este puede ser el caso, aun cuando mejore la balanza en términos de moneda extranjera, pues la magnitud de un determinado déficit extranjero, medido en moneda nacional, aumentará como resultado de la devaluación.

Díaz-Alejandro (1963) y (1965) destaca los efectos redistributivos asociados a una devaluación. En términos generales, una devaluación in. ducirá una redistribución del ingreso desde el sector de los bienes no negociables, hacia aquellos que se encuentran en el sector de los bienes negociables. El efecto neto de esta redistribución del ingreso en el gasto interno total, dependerá de la magnitud relativa de la propensión margi. nal al gasto que tengan tanto los perdedores como los ganadores. DíazAlejandro comenta en forma convincente, que la redistribución del in. greso de los trabajadores hacia los terratenientes que resultó de la devaluación del peso argentino a fines de 1958, originó una aguda recesión al año siguiente. Aparentemente, en Finlandia ocurrió algo semejante después de la devaluación de 1957 (ver A. Gerakis (1964))

P. Krugman y L. Taylor (1978) recientemente formalizaron los efectos anteriores en un modelo de tipo keynesiano de una economía abierta, agregando el "efecto fiscal" que induce la redistribución del ingreso del sector privado hacia el sector público, hecho que ocurre frecuentemente después de una devaluación. Si hay impuestos ad valorem sóbre las exportaciones o las importaciones, el incremento de precio de los bienes negociados redistribuirán el ingreso hacia el gobierno; también, si existen impuestos progresivos sobre la renta o impuestos mayores sobre aquellos ingresos que no sean salarios, el aumento nominal del ingreso significará un incremento proporcional más elevado que irá a manos del Estado.

Los efectos deflacionarios mencionados se refieren al impacto de la devaluación sobre el gasto global interno, y más exactamente, sobre el consumo interno, al reducir el ingreso disponible y/o del valor del multiplicador que resulte de los efectos redistributivos. Aunque anteriormente 
no se introdujo explícitamente como componente de la demanda global, su posible declinación durante la contracción económica originada por la devaluación, lógicamente se consideraría en estos modelos como el resultado - no la causa - de la reducción en el consumo. Sin embargo, en el caso de México, como se indicó en la sección anterior, la aguda reducción de las inversiones aparentemente tuvo una función primordial para precipitar la recesión de 1976-77. Por lo tanto, surge la necesidad de investigar más a fondo la posibilidad de un efecto directo de la devaluación sobre las decisiones de inversión de una empresa - posibilidad que la literatura aparentemente aún no reconoce plenamente.

\subsection{Los efectos de una devaluación sobre las decisiones de inversión de una empresa}

\subsubsection{Devaluación anticipada versus devaluación no anticipada}

Como se comentó en la primera parte de este estudio, para 1973-1974 era ya evidente la sobrevaluación del peso. Conforme transcurría el tiempo, crecía la posibilidad de una devaluación; pronto, sólo fue cuestión de cuándo y cuánto se despreciaría el peso frente al dólar. El público en su mayoría, tanto familias como empresas, deben haber tenido expectativas respecto de la oportunidad y magnitud de la posible devaluación; esto debió influir en su comportamiento. Por lo tanto, sería erróneo no efectuar el análisis de las expectativas al estudiar los efectos de la devaluación del peso.

En la medida en que una empresa anticipa correctamente la devaluación, es obvio que cuando ésta ocurra, no surtirá efecto sobre aquélla: la empresa habrá ajustado completamente sus decisiones al momento de revisar en detalle sus expectativas. Sólo la porción no anticipada de la depreciación de la moneda tendrá efectos reales en el momento que ocurra.

En el caso de México, es conveniente analizar con más detalle algunos aspectos de la formación de las expectativas. En lo que respecta a los tipos de cambio flexibles, una suposición común que se hace en la literatura es, que el cambio esperado en la paridad, medida por la prima de descuento en los mercados a futuro, es proporcional a la diferencia entre una "tasa de equilibrio a largo plazo" y la tasa de contado corriente. Este tipo de cambio a largo plazo, normalmente se determina de acuerdo con alguna versión del teorema de la paridad del poder adquisitivo. ${ }^{18} \mathrm{Sin}$ embargo, respecto de los tipos de cambio fijos, no existe tal consenso. Cuando sobrevino la devaluación de 1976, hacía 22 años que el peso estaba ligado al dólar, aunque desde 1973 se descontaba en el mercado de futuros, cuando este último se estableció en Chicago. El hecho que una devaluación fuera una decisión discrecional de las autoridades monetarias,

18 Véase, por ejemplo, R. Dornbusch (1976). 
permitió que las expectativas se vieran frustradas continuamente, sin implicar irracionalidad por parte de quienes participaban en el mercado: en el caso de las paridades fijas, las expectativas no se basaban tanto en el poder adquisitivo de la moneda en términos de bienes, cuanto en la predicción de la decisión gubernamental de devaluar.

Además, tan pronto se reconoce que la prima de descuento no es un mecanismo de predicción imparcial de la evolución futura del tipo de cambio, se torna evidente que no es lo óptimo cubrir en su totalidad de las transacciones corrientes en moneda extranjera; por lo tanto, la cobertura sólo parcial de obligaciones en moneda extranjera no implica un comportamiento irracional por parte del sector privado.

Considérese el problema de decisión por parte de un agente económico (una empresa) respecto de la cobertura óptima a futuro de las obligaciones pendientes contratadas (denominadas) en moneda extranjera. Consideremos que el agente minimiza el valor actual de los costos esperados de conservar obligaciones extranjeras (o la esperada desutilidad de los costos) asociados con la posibilidad de una devaluación, $u(c), y$ decide sobre la proporción de estas obligaciones, $\delta$, que deben cubrirse. Formalmente, el problema puede escribirse de la siguiente manera:

$$
\operatorname{Min}_{\delta} \int_{0}^{\infty} E(u(c)) e^{-r t} d t
$$

Si $x L^{f}$ es el total de las obligaciones extranjeras pendientes en términos de moneda nacional ( $x=$ tipo de cambio, $L^{t}=$ préstamos externos), los costos totales son:

$$
c=\left\{\begin{array}{l}
\delta d x L^{f} \text { antes de la devaluación } \\
(1-\delta) x^{\prime} \hat{x} L^{f} \text { el día de la devaluación. }
\end{array}\right.
$$

El término superior de (1) representa el costo de cubrir una proporción $\delta$ de obligaciones pendientes, que se aproxima por el descuento a futuro $d$. El término inferior refleja el costo de la parte no cubierta de las obligaciones extranjeras si ocurre la devaluación, el cual, por definición, es igual a la pérdida cambiaria sufrida: $x^{\prime} \hat{x} L^{f}$, en donde $x^{\prime}$ es el nuevo tipo de cambio y $\hat{x}$ es el cambio porcentual de $x .^{19}$ Si $x$ se distribuye de acuerdo con un proceso de Poisson con parámetro $\lambda$, el problema anterior podrá formularse así:

$$
\operatorname{Min}_{\delta} \int_{0}^{\infty}\left\{e^{-\lambda t} u\left(\delta d x L^{f}\right)+\lambda e^{-\lambda t} u\left((1-\delta) x^{\prime} \hat{x} L^{f}\right)\right\} e^{-r t} d t
$$

19 Con fines de concisión, consideramos que $\hat{x}=d$, de modo que no se sufre pérdida en el cambio en la porción cubierta de las obligaciones externas. 
La condición de primer orden para un mínimo es:

$$
d x L^{\dagger} u^{\prime}\left(\delta d x L^{f}\right)-\lambda x^{\prime} L^{\dagger} u^{\prime}\left((1-\delta) x^{\prime} \hat{x} L^{f}\right)=0
$$

y resolviendo para $\delta$ obtenemos, bajo el supuesto que $u(c)=\log c$ :

$$
\delta=\frac{1}{1+\lambda}
$$

Parece razonable considerar que $\lambda$, que es el parámetro de distribución, depende del descuento a futuro $d: \lambda(d), \lambda^{\prime}<0$. Conforme aumenta el descuento, también aumenta la probabilidad de que ocurra la devaluación en algún momento $t$ más cercano al presente, y el comportamiento óptimo de la empresa según la ecuación (2) es cubrir una proporción mayor de sus obligaciones.

Si las empresas se comportan de acuerdo con el criterio de racionalidad descrito en la formalización anterior, y si la mayor parte de la cobertura de futuros es realmente efectuada por las mismas, observaríamos una relación directa entre el tamaño del descuento y el volumen de contratos negociados en el mercado de futuros. Comprobamos esta relación por el período abril/septiembre de $1976,{ }^{20}$ utilizando la siguiente ecuación:

$$
1 n F C_{t}=a_{o}+a_{1} 1 n F D_{t}^{T}+u_{t}
$$

en donde:

$$
\begin{aligned}
& F C_{t}=\begin{array}{l}
\text { promedio semanal de los contratos a futuro nego- } \\
\text { ciados, }
\end{array} \\
& F D_{t}^{T}=\begin{array}{l}
\text { promedio semanal de la prima a futuro de los con- } \\
\text { tratos que vencen en } T .
\end{array}
\end{aligned}
$$

Los resultados son: (3) ${ }^{22}$

20 Las cotizaciones diarias sobre el número de contratos negociados en el mercado de futuros de Chicago aparecieron en el Wall Street Journal (que fue nuestra fuente), sólo desde abril de 1976. No intentamos reunir datos anteriores pues aparentemente este es el período más importante para nuestros propósitos.

21 Los datos disponibles respecto del número de transacciones no se separó de acuerdo con el vencimiento del contrato. Es decir, no podemos decir si un contrato negociado en un cierto día vence en septiembre o diciembre de 1976. Sin embargo, dado que la prima de descuento para fechas diferentes se mueve parejo en la misma dirección, no necesitamos hacer ningún supuesto restrictivo respecto de la distribución de los contratos. Esto se evidencia por los resultados que se presentan arriba: la magnitud de los coeficientes y las demás características de las dos regresiones son muy similares.

22 Las cifras entre paréntesis son estadísticas $t$. 


$$
\begin{aligned}
& 1 n F C_{t}=7.03+.431 n F D_{t}^{\text {sep } 76} \\
& \text { (30.3) (6.7) } \\
& R^{2}=.68 \\
& \text { D.W. }=2.1 \\
& 1 n F C_{t}=7.47+.741 n F D_{t}^{d e c} 76 \\
& \text { (26.8) (6.7) } \\
& R^{2}=.70 \\
& \text { D.W. }=2.1
\end{aligned}
$$

Los coeficientes tienen los signos esperados, son significativos, y la estadística de Durbin-Watson no muestra autocorrelación.

\subsubsection{La devaluación y las decisiones de inversión}

Continuando con la discusión anterior, distinguiremos tres efectos de una devaluación que, en el caso de México afectan la decisión de las empresas respecto de sus inversiones:

a) un efecto-riqueza

b) un efecto-liquidez

c) un efecto de reestructuración de pasivos.

a) El efecto-riqueza

Ocurre el efecto-riqueza si alguna perturbación que afecta el balance de un agente económico, cambia su patrimonio. En el caso de una empresa - a la que deseamos considerar como agente económico en su propio derecho independiente de sus propietarios - ${ }^{23}$ una devaluación puede producir un efecto-riqueza, en la medida en que difiera el sobreprecio o la depreciación de activos y pasivos que resulte de la misma.

Asimismo, una devaluación afecta las obligaciones pendientes no cubiertas contratadas en moneda extranjera en términos de moneda nacional. Por el lado de los activos, una devaluación tiene un efecto directo sobre aquellos activos cuyos precios hayan sido fijados internacionalmente; es decir, los "activos negociables". Aunque el efecto-riqueza puede modificar el patrimonio de una empresa en cualquier dirección, ${ }^{24}$ existe una asime-

23 Dentro de un marco de equilibrio general no existe un efecto de riqueza como tal a nivel de la empresa. Cualquier cambio en el patrimonio de la firma es sólo un reflejo de algún cambio en el patrimonio de los accionistas y su importancia se reduce a un efecto-riqueza tradicional sobre las decisiones de consumo de las familias.

24 Para una sola empresa, el efecto neto dependerá del tamaño de la deuda externa no cubierta en relación con el tamaño de los activos negociables. Si una empresa ha adquirido activos negociables con crédito doméstico y tiene una exposición neta en moneda extranjera igual a cero, una devaluación incrementará su 
tría en la práctica que tiende a desviar este efecto en contra de la empresa. La revaluación de las obligaciones es automática, en tanto que la de los activos depende tanto de consideraciones legales como de mercado. En el caso de México, no se autorizó a la mayoría de las empresas la revaluación de los activos para fines legales (y contables).

Es razonable considerar que para una empresa el costo de financiamiento está en relación inversa a su patrimonio. Esta consideración se apoya en dos argumentos. Primero, cuanto mayor sea el patrimonio de una firma, menor será el riesgo de incumplimiento, desde el punto de vista bancario. Segundo, podría darse el caso de que en un mercado menos que perfecto, la posición de ajuste de una empresa afecte el precio al que puede emitir nuevas acciones. La conclusión es, que un efectoriqueza negativo (patrimonio decreciente) afectará las decisiones de inversión de una empresa dado el impacto que tiene sobre los costos de financiamiento.

\section{b) El efecto-liquidez}

Aparece el efecto-liquidez si alguna perturbación genera una diferencia entre la disponibilidad de dinero real y la deseada, y si el agente económico no puede regresar, sin costo extra, a su posición preferida de liquidez.

Después de una devaluación una empresa puede tener una diferencia entre la disponibilidad de dinero real y deseado por diversas razones. Primero, el costo en moneda nacional del servicio de la deuda experimenta un aumento inmediato y discontinuo. Segundo, la cuenta que ampara materias primas importadas y posibles ajustes salariales, puede elevarse. Tercero, si ocurre un efecto-riqueza y/o las autoridades adoptan políticas monetarias restrictivas, el costo del servicio de la deuda interna también puede elevarse. El incremento que se produce en los pagos corrientes de la empresa exigirá saldos operativos superiores.

Por otra parte, es probable que las empresas no puedan reconstruir de inmediato sus saldos en efectivo sin incurrir en costos más elevados. Primero, los activos no líquidos no pueden venderse inmediatamente al precio total que tienen en el mercado; segundo, y tal vez más importante, en el caso que estudiamos, es que las restricciones del mercado crediticio implican un incremento en las tasas de interés acorde con el flujo de empréstitos.

patrimonio. Por otra parte, si la empresa ha contratado deudas externas para financiar gastos corrientes o activos no negociables, la devaluación reducirá su patrimonio. Es probable que la situación de la mayoría de las empresas haya quedado entre estos dos casos polares. Para todas las empresas el efecto neto dependerá del tamaño de la deuda extranjera privada en relación con el tamaño de las importaciones anteriores acumuladas de bienes de capital, la tasa de depreciación vigente en el mercado y el grado de sustituibilidad entre bienes de inversión nacionales e importados. 
Una empresa que tiene problemas de liquidez, deberá dedicar recursos para volver a reconstituir su capital de trabajo antes de decidir emprender proyectos de inversión.

\section{c) El efecto-reestructuración de obligaciones}

La creciente incertidumbre acerca del valor futuro del tipo de cambio provocado por el abandono de una paridad fija, puede inducir a las empresas a restructurar sus obligaciones; es decir, a reintegrar o sustituir préstamos pendientes en moneda extranjera. No puede determinarse a prio$r i$ si la incertidumbre repecto al tipo de cambio aumenta o disminuye después de una devaluación, ya que ello depende de las expectativas del público.

En el caso de México, como se sugirió anteriormente, el tipo de incertidumbre prevaleciente antes de la devaluación estuvo más relacionado con la oportunidad de la decisión gubernamental para modificar el tipo de cambio, que con su magnitud. Sin embargo, una vez operada la devaluación, la incertidumbre se desplazó hacia la inestabilidad del tipo de cambio. Bien pudo haber sido el caso que la incertidumbre aumentara después de agosto de $1976 .{ }^{25}$ Considerando las dificultades que enfrentaron las empresas para obtener la cobertura adecuada a futuro, el riesgo adicional pudo haberlas obligado a restructurar sus obligaciones.

$\mathrm{Si}$ la tasa de interés interna aumentara con el flujo de empréstitos (debido a las restricciones en el mercado crediticio ya mencionadas), la empresa tendría que reducir sus inversiones utilizando los fondos disponibles para reintegrar sus deudas extranjeras.

\subsubsection{El modelo}

Consideremos el problema de decisión que encara una empresa que produce un solo producto inmediatamente después de la devaluación. Formalizaremos este problema dentro de un marco cronológico discreto y consideraremos que el objetivo de la empresa es maximizar las ganancias es-

25 Consideramos que antes de la devaluación, la incertidumbre tuvo la siguiente estructura: primero, el instante de la devaluación se distribuyó de acuerdo con el proceso de Poisson con un parámetra $\lambda$; segundo, que el valor del tipo de cambio después de abandonar la paridad fija se distribuyó de acuerdo a una distribución normal con una media $\hat{x}$ y varianza $\sigma^{2}$. También consideramos que una vez operada la devaluación, el tipo de cambio esperado prevaleciente en el siguiente período, se distribuyó de acuerdo con una distribución normal con una media $\hat{x}^{\prime}$ y varianza $\sigma^{2}$. Entonces hubiera sido fácil, para una función determinada de utilidad (definiendo la aversión a los riesgos, de la firma), anotar una relación explícita entre los cinco parámetros introducidos anteriormente, expresando la condición bajo la cual habría aumentado el riesgo percibido. 
peradas al final del período. Al efecto introducimos las siguientes variables:

$$
\begin{aligned}
x_{0} & =\text { tipo de cambio al comienzo del período (c.d.p.) } \\
x & =\text { tipo de cambio al final del período (f.d.p.) } \\
\pi_{o} & =\text { utilidades retenidas al c.d.p. } \\
\pi(x) & =\text { utilidades al f.d.p. } \\
p^{*} & =\text { precio esperado de la producción al f.d.p. } \\
\bar{w} & =\text { nivel de los salarios durante el período } \\
N & =\text { cantidad de mano de obra empleada durante el período } \\
K_{o} & =\text { capital social al c.d.p. } \\
I & =\text { inversiones durante el periodo } \\
K & =\text { capital social al f.d.p. } \\
L_{o}^{d} & =\text { créditos pendientes en moneda nacional al c.d.p. } \\
l^{d} & =\text { créditos solicitados durante el período } \\
L^{d} & =\text { créditos pendientes en moneda nacional al f.d.p. } \\
L_{o}^{t} & =\text { créditos pendientes en moneda extranjera al c.d.p. } \\
l^{t} & =\text { créditos extranjeros solicitados durante el período } \\
L^{f} & =\text { créditos pendientes en moneda extranjera al f.d.p. } \\
L_{o} & =\text { obligaciones totales pendientes al c.d.p. } \\
L & =\text { obligaciones totales pendientes al f.d.p. } \\
M_{o} & =\text { disponibilidad monetaria al c.d.p. } \\
m & =\text { adiciones a la disponibilidad monetaria durante el período } \\
M & =\text { disponibilidad monetaria al f.d.p. } \\
i_{d} & =\text { tasa de interés interna } \\
i_{f} & =\text { tasa de interés externa }
\end{aligned}
$$

Procederemos a hacer las siguientes suposiciones:

A0: No se pueden emitir acciones ni se permite cobertura extranjera a futuro. Esta doble consideración capta, de forma aproximativa, las características imperfectas tanto del mercado de acciones como de los mercados cambiarios a futuro. Por tanto, las únicas variables de decisión de las empresa son $N, l^{d}$ y $l^{t}$.

A1: Los pagos de intereses se calculan en base a las obligaciones pendientes al final del período.

A2: Los créditos extranjeros solicitados durante el periodo pueden convertirse en moneda nacional al tipo de cambio vigente, al comienzo del período.

A3: La tasa de interés interna es una función creciente tanto de la proporción deuda/capital al comienzo del período, como del flujo de créditos internos durante el período. La tasa de interés externa es una función creciente de la proporción deuda/capital al comienzo del período. Por tanto, estamos considerando que prevalecen restricciones cuantitativas en los mercados crediticios internos, en tanto 
que los costos crecientes de los créditos extranjeros sólo se deben a consideraciones del riesgo tal como las perciben los banqueros extranjeros. Suponemos que estas funciones son lineales.

A4: La disponibilidad monetaria deseada es proporcional a los desembolsos esperados por concepto de salarios y pago de intereses al final del período, y aumentan de acuerdo con la varianza de estos desembolsos.

A5: El tipo de interés al final del período, como lo percibe la empresa, se distribuye de acuerdo con una distribución normal con una media $\hat{x}$ y varianza $\sigma^{2}$.

A6: La función de la utilidad de la empresa, $u(\cdot)$, es cuadrática.

A7: La función de la producción, $f(\cdot)$, presenta las propiedades neoclásicas usuales.

De acuerdo con las consideraciones anteriores, el problema de la decisión para la empresa puede formalizarse de la siguiente manera: ${ }^{26}$

Máx $V\left(N, l^{d}, l^{f}\right)=p^{*} f(K, N)-\bar{w} N-i_{d} L^{d}-i_{f} \bar{x} L^{f}-\rho \sigma^{2}\left(i_{f} L^{f}\right)^{2}$

en donde

$$
N, l^{d}, l^{t}
$$

$$
\begin{aligned}
& K=K_{o}+I \\
& I=\pi_{o}+l^{d}+x_{o} l^{f}-m \\
& m=\alpha \bar{w} N+\beta\left(i_{d} L^{d}+i_{f} \bar{x} L^{f}\right)+\gamma \sigma^{2} i_{f} L^{f}-M_{\mathrm{o}} \\
& L^{d}=L_{o}^{d}+l^{d} \\
& L^{f}=L_{o}^{f}+l^{f} \\
& i_{d}=\bar{\imath}_{d}+a \frac{L_{o}}{K_{o}}+b \frac{l^{d}}{K_{o}} \\
& i_{f}=\bar{\imath}_{f}+c \frac{L_{o}}{K_{o}}
\end{aligned}
$$

Las condiciones de primer orden para un máximo son:

$$
\frac{\partial V}{\partial N}=p^{*} \frac{\partial f}{\partial N}-\bar{w}\left(l+\alpha \frac{\partial f}{\partial N}\right)=0
$$

26 Es bien sabido que bajo los supuestos A5 y A6 la hipótesis de utilidad esperada es equivalente al análisis de varianza. Eso es, podemos sustituir, como función objetivo del problema, $\int_{-\infty}^{\infty} u[\pi(x)] d x$ por $E[\pi(x)]-p$ var $[\pi(x)]$, en donde $\rho$ es la medida de la aversión al riesgo absoluto. 


$$
\begin{gathered}
\frac{\partial V}{\partial l^{d}}=p^{*} \frac{\partial f}{\partial K}\left[1-\beta\left(\bar{l}_{d}+a \frac{L_{o}}{K_{o}}+b \frac{l^{d}}{K_{o}}\right.\right. \\
\left.\left.+\frac{b L^{d}}{K_{o}}\right)\right]-\left(\bar{l}_{d}+a \frac{L_{o}}{K_{o}}+b \frac{l^{d}}{K_{o}}+\frac{b L^{d}}{K_{o}}\right)=0 \\
\frac{\partial V}{\partial l^{t}}=p^{*} \frac{\partial f}{\partial K}\left[x_{o}-i_{f}\left(\beta \bar{x}+\gamma \sigma^{2}\right)\right]- \\
i_{f} \bar{x}+2 \rho \sigma^{2} i_{f}\left(L_{o}^{t}+l^{f}\right)=0
\end{gathered}
$$

Resolviendo las ecuaciones (4) y (5) para los valores $l^{d}$ y $l^{t}$ obtenemos:

$$
\begin{aligned}
& l^{d}=\frac{K_{o}}{2 b}\left(\frac{p^{*}}{1+\beta p^{*} \frac{\partial f}{\partial K}} \frac{\partial f}{\partial K}-\vec{i}_{d}-a \frac{L_{o}}{K_{o}}\right)-\frac{L_{o}^{d}}{2} \\
& l^{\prime}=\frac{1}{2 \rho \sigma^{2} i_{f}^{2}}\left[\left(x_{o}-\left(\beta \bar{x}+\gamma \sigma^{2}\right) i_{f}\right) p^{*} \frac{\partial f}{\partial K}-\right. \\
& \left.i_{f} \bar{x}\right]-L_{o}^{f}
\end{aligned}
$$

Ambas condiciones tienen una clara interpretación económica. Expresan el hecho de que el nivel óptimo del crédito neto recibido, tanto en moneda nacional como en moneda extranjera, se determina de tal modo que se igualan los costos marginales respectivos. En el caso de los créditos recibidos en moneda nacional, un préstamo marginal de un peso representa un costo de $i_{d}+\frac{b}{K_{o}} L^{d}$ y produce un beneficio, $p^{*} \frac{\partial f}{\partial K} \frac{1}{1+\beta p^{*} \frac{\partial f}{\partial K}}$,

igual a la productividad marginal del capital, multiplicado por la proporción del préstamo marginal que puede invertirse en forma productiva después de deducir los saldos operativos de compensación; por lo tanto, resulta la condición (5). En el caso de los créditos extranjeros, un préstamo marginal de un dólar representa un costo de intereses directo de $\bar{x} i_{f}$ y un costo indirecto, $2 \rho \sigma^{2} i_{f} L^{t}$, debido a la creciente incertidumbre; reditúa un beneficio, $p^{*} \frac{\partial f}{\partial K}\left(x_{o}-\left(\beta \bar{x}+\gamma \sigma^{2}\right) i_{f}\right)$, igual a la productividad marginal del capital multiplicada por la fracción del préstamo marginal que puede invertirse en forma productiva después de deducir los saldos operativos de compensación: de ahí tenemos la condición (6). 
Sustituyendo (6) y (7) en la definición de la inversión, finaimente obtenemos:

$$
\begin{aligned}
I & =\pi_{o}+M_{o}-\alpha \bar{w} N+\frac{K_{o}\left(1-\beta i_{d}\right)}{2 b}\left(\frac{p^{*}}{1+\beta p^{*} \frac{\partial f}{\partial K}} \frac{\partial^{f}}{\partial K}-\bar{i}_{d}\right) \\
& +\frac{x_{o}-i_{f}\left(B \bar{x}+\gamma \sigma^{2}\right)}{2 \rho \sigma^{2} i_{f}^{2}}\left[\left(x_{o}-\left(\beta \bar{x}+\gamma \sigma^{2}\right) i_{f}\right) p^{*} \frac{\partial f}{\partial K}-\overline{x l_{f}}\right] \\
& -\frac{1}{2}\left[1+\frac{a}{b}+\left(1-\frac{a}{b}\right) \beta i_{d}+\frac{x_{o}-i_{f}\left(\beta \bar{x}+\gamma \sigma^{2}\right)}{\rho \sigma^{2} i_{f}^{2}} \frac{c \bar{x}}{K_{o}}\right] L_{o}^{d} \\
& -\frac{1}{2}\left[2+\frac{a}{b}-\frac{a}{b} \beta i_{d}+\frac{x_{o}-i_{f}\left(\beta \bar{x}+\gamma \sigma^{2}\right)}{\rho \sigma^{2} i_{f}^{2}} \frac{c \bar{x}}{K_{o}}\right] x_{o} L_{o}^{f}(8)
\end{aligned}
$$

Nótese que $i_{d}$ no se expresó arriba en términos de su definición (incluyendo $l^{d}, L_{o}$ y $\left.K_{o}\right)$ siempre que aparezca multiplicado por $\beta$, que supuestamente es "pequeña" respecto de 1 . Asimismo, $i_{f}$ no fue sustituida por su definición, $\bar{\imath}_{f}+c \frac{L_{o}}{K_{o}}$; siempre que aparezca al cuadrado (la variabilidad de $i_{f}^{2}$ es de magnitud de segundo orden respecto de la variabilidad de $i_{f}$, si $c$ es "pequeña") o multiplicada por $\beta$ o $\gamma$ suponiendo que $\gamma$ también sea "pequeña" respecto de 1.

La ecuación (7) tiene la interpretación económica siguiente: El modelo desarrollado anteriormente corresponde básicamente a una formalización de tipo Jorgenson ${ }^{27}$ del problema de inversión de la empresa, en el cual se incorporó una estructura financiera detallada. Ya que consideramos que la firma encara costos de financiamiento con crecimiento acelerado, parece razonable suponer que los costos reales de la inversión aumentarán a un ritmo relativamente más lento: entonces podemos enmarcar el ajuste a un capital social "deseado" que pueda originarse dentro del período que se estudia. En la tradición de Jorgenson, las inversiones se efectúan debido a una discrepancia entre la productividad marginal del capital y su costo de arriendo, que está determinado por la tasa de interés.

Sin embargo, en nuestro modelo el costo de arriendo del capital no es una simple tasa de interés, dado que capta las relaciones financieras que se elaboraron en el modelo. Debido a los costos crecientes de financiamiento externo, el nivel de la inversión será superior, mientras el nivel de utilidades retenidas sea $\left(\pi_{o}\right)$. Debido a los costos de liquidez, cuanto mayores sean los saldos monetarios no comprometidos $\left(M_{o}-\alpha \bar{w} N\right)$, mayor será el nivel de la inversión. Los términos cuarto y quinto reflejan discrepancias entre el valor del producto marginal del capital y el com-

27 Ver D. Jorgenson (1963). 
ponente fijo del costo de financiamiento: claramente resulta que mientras más alta sea la restricción crediticia en el mercado nacional (medido por $b$ ), mayor sea la incertidumbre cambiaria y el grado de aversión por el riesgo (medidos por $\sigma$ y $\rho$, respectivamente), entonces serán más fuertes los efectos-liquidez y sustitución de pasivos. En consecuencia el nivel de inversión será menor. Finalmente, los dos últimos términos engloban el efecto negativo en la inversión del tamaño de los pasivos iniciales: mientras mayores sean las obligaciones, más fuertes serán los tres efectos mencionados.

\section{Resultados empíricos}

A partir de la ecuación (8), podemos derivar una ecuación estimativa simplificada que podemos representar así:

$$
I=a_{o}+a_{1} \pi_{o}+a_{2} M_{o}+a_{3} K_{o}+a_{4} L_{o}^{d}+a_{5} L_{o}^{f}
$$

Esta derivación se basa en tres suposiciones: Primero, consideramos los coeficientes de comportamiento del modelo iguales para todas las empresas (es decir, $\alpha, \beta, \gamma, \rho, \sigma, a, b, c, \bar{l}_{d}$ e $\bar{l}_{f}$ ). Segundo, consideramos $i_{d}$ e $i_{f}$ iguales para todas las empresas siempre que aparezcan en términos de magnitud de segundo orden. Tercero, y más importante, consideramos que por el momento la productividad marginal esperada del capital, $p^{*}$ $\frac{\partial f}{\partial K}$, es constante y uniforme para todas las firmas; aunque esto parece una suposición restrictiva (dada la aparente diversidad de condiciones económicas en los diferentes sectores industriales) puede argüirse que un "pesimismo" muy extendido, reflejado por $p^{*}$, absorbió la mayor parte de la diversidad sectorial en $\frac{\partial f}{\partial K}$ durante el período bajo estudio. Además, a este nivel, suprimimos la variable salarial debido a las restricciones en la información. Sin embargo, también puede argüirse que a corto plazo la proporción capital/mano de obra es constante de modo que es posible fijar $\bar{w} N$ como una proporción de $K$ y por tanto incorporar el término salarial en el coeficiente $a_{3}$.

Estimamos la ecuación (9) utilizando una encuesta con los balances y estados de pérdidas y ganancias de 121 empresas grandes. ${ }^{28}$ En este do-

28 La encuesta consistió en un primer cuestionario de inversiones "cualitativas" y una segunda parte en la cual las empresas daban a conocer sus balances y estados de ingresos. De las 1999 empresas encuestadas, sólo 764 proporcionaron ambos documentos, que después fueron verificados en concordancia e integridad. En base a este criterio, se escogió una muestra de 121 empresas: esas empresas no sólo presentaron documentos detallados, sino que aparentemente eran más congruentes entre sí. 
cumento mostramos algunos resultados preliminares (I corresponde a la inversión durante 1977 y el índice 0 se refiere a diciembre de 1976).$^{29}$

$$
\begin{aligned}
& I=4422.9+.04 \pi_{o}+.07 M_{o}+.19 K_{o}-.24 L_{o}^{d}-.15 L_{o}^{f} \\
& \text { (.9) } \\
& \text { (.2) } \\
& \text { (.9) } \\
& \text { (3.2) } \\
& (-2.7) \quad(-1.9) \\
& R^{2}=.11 \\
& \text { Estadísticas } F=2.94 \\
& \text { NN de observaciones: }
\end{aligned}
$$

Observamos que, aunque todos los coeficientes tienen los signos esperados, sólo tres de ellos son significativos. El coeficiente de $K_{0}$ capta en forma significativa un efecto de escala positiva; los coeficientes de los niveles de créditos internos y externos son significativamente negativos, apoyando de esta manera nuestra hipótesis básica acerca del efecto de la devaluación sobre la inversión a través del pasivo de los balances de las empresas. Por el lado de los activos de las firmas, sin embargo, el coeficiente de $M_{\circ}$ y de $\pi_{o}$ es mucho menor a uno y no es significativo.

Este resultado puede deberse a diversas razones. Primera, nuestra medida de la posición de liquidez de una empresa incluyó los inventarios al comienzo del período; el valor inferior del coeficiente $M_{o}$ puede captar el efecto negativo de inventarios altos en las expectativas de la empresa y sus decisiones de inversión. Segunda, nuestra medida de las utilidades corresponde a las utilidades retenidas durante 1976; claramente, una medida de las utilidades de 1977 sería un mejor sustituto para la variable que requiere el modelo teórico, dado que las utilidades de 1976 ya fueron repartidas.

Después intentamos emplear medidas más adecuadas para $M_{o}$ y $\pi_{o}$ a fin de mejorar la estimación de los coeficientes correspondientes. Sin embargo tuvimos que restringir más aún el número de empresas de nuestra muestra, ya que fueron menos las empresas que proporcionaron estimaciones semestrales de las utilidades y tenencias monetarias. El resultado que se obtuvo fue el siguiente:

$$
\begin{array}{r}
I=\underset{(.05)}{-914.2}+\underset{(.24)}{.03 \pi_{1}}+\underset{(2.5)}{2.4 M_{1}}+\underset{(1.43)}{.12 K_{o}-.03 L_{o}^{d}-2.9 L_{o}^{\dagger}} \\
(-1.40)(-1.71) \\
R^{2}=.24 \\
\text { Estadísticas } F=3.6 \\
\text { No de observaciones: }
\end{array}
$$

$M_{1}$ corresponde a los promedios de los saldos en efectivo reportados en junio y diciembre de 1976 , en tanto que $\pi_{1}$ corresponde al promedio de utilidades obtenidas en 1977. De nuevo, los coeficientes tienen todos el

20 Las estadísticas $t$ aparecen entre paréntesis. 
signo correcto, aunque los que corresponden a $K_{o}$ y $L_{o}^{d}$ sólo son significativos a un nivel de $10 \%$. El coeficiente de $M_{1}$ fue muy alto y significativo, pero aún no pudimos mejorar el coeficiente de $\pi_{1}$ a pesar del cambio de definición. Estas estimaciones, repetimos, son preliminares, dado que aún falta mucho trabajo para eliminar incongruencias en los datos que sirvieron de base.

Finalmente, estimamos la ecuación (9) en primeras diferencias, utilizando el cambio de inversión entre 77 y 76 como variable dependiente:

$$
\begin{aligned}
& \Delta I_{77 / 76}=\underset{(1.65)}{26189.4}+\underset{(0.3)}{.008 \Delta \pi}+\underset{(2.03)}{.97 \Delta M}-\underset{(-1.64)}{.62 \Delta L^{d}-.53 \Delta L^{f}} \\
& R^{2}=.37 \\
& \text { Estadísticas } F=2.9 \\
& \text { No de observaciones: }
\end{aligned}
$$

De nuevo, todos los coeficientes resultaron con los signos esperados, aunque los que corresponden a $L^{d}$ y $L^{t}$ sólo son significativos a un nivel de $10 \%$.

\section{Conclusiones y ampliaciones}

Subrayamos en este documento cuatro puntos: Primero, que una devaluación puede tener efectos deflacionarios importantes a corto plazo por su impacto sobre las decisiones de inversión de las empresas, tal como se desprende de nuestro estudio de la devaluación del peso en México en 1976.

Segundo, la literatura existente sobre el tema de la devaluación, incluso las contribuciones que tratan sus aspectos deflacionarios, no incluye estos efectos. Tercero, consideramos que los tres efectos que se analizaron en la sección 2 (riqueza, liquidez y sustitución de obligaciones) corresponden a los principales canales a través de los cuales una devaluación ejerce su influencia deflacionaria sobre la inversión. Cuarto, nuestras estimaciones empíricas, aunque preliminares, parecen apoyar nuestra tesis.

La severidad de los efectos deflacionarios de una devaluación sobre la inversión, dependen tanto del grado de apertura financiera de la economía, como de la relativa sofisticación de las instituciones financieras nacionales. En el caso de México, los estrechos lazos del sector privado con las instituciones de crédito estadounidenses, el subdesarrollo del mercado de divisas a futuro y la imperfección de los mercados financieros nacionales (es decir, el reducido tamaño de las negociaciones de acciones) cré un ámbito original y amplificó la magnitud de la caída de la inversión ocasionada por la devaluación. En este sentido, la devaluación de México constituye un caso especialmente interesante de estudio. His- 
tóricamente, la mayoría de las devaluaciones importantes han ocurrido en países en desarrollo, en los cuales las empresas privadas no habían contraído deudas de tamaño significativo en moneda extranjera; por otra parte, la flexibilidad en los tipos de cambio entre las economías industriales integradas financieramente evitó que los efectos deflacionarios en la inversión alcanzaran una magnitud apreciable en términos empíricos. Esta es quizá la razón por la cual, la literatura sobre devaluación no ha reconocido la posibilidad teórica de estos efectos ni su creciente importancia para los países en desarrollo que poco a poco se integran a los mercados financieros internacionales.

Ese documento podría ampliarse en las siguientes direcciones.

Primero, el modelo aquí desarrollado puede transformarse a fin de que comprenda los efectos de la devaluación en los costos y precios de las empresas, haciendo que el nivel de las utilidades se convierta en variable endógena. Queda claro que puede ocurrir un efecto deflacionario secundario en la inversión, si las empresas tienen que absorber parcialmente los costos crecientes (de materias primas importadas y salarios) con márgenes de utilidad reducidos.

Segundo, podríamos investigar la magnitud empírica de los efectos más tradicionales de la devaluación en el caso de México: el efecto-riqueza negativo sobre el consumo, el comportamiento de las balanzas comercial y de pagos, y los aspectos redistributivos de los ajustes cambiarios.

Finalmente, parece interesante ahondar más en el proceso de sustitución monetaria o "dolarización" que siguió a la devaluación mexicana. Si la demanda de moneda nacional disminuye (o se desestabiliza), la oferta monetaria excedente que existía antes de la devaluación, según la visión monetarista, no se absorberá conforme disminuya la oferta de saldos reales con los ajustes del tipo de cambio y de precios; así, la devaluación no podrá corregir los desequilibrios en los mercados de activos. Las implicaciones de estos fenómenos en torno a la conducta de la política monetaria son obvios: no es fácil para un país en desarrollo seguir una flotación independiente y conservar la libre convertibilidad monetaria. En el caso de México, las autoridades tuvieron que reforzar su intervención en el mercado cambiario (para evitar las grandes fluctuaciones en la paridad entre el peso y el dólar) al punto que se adoptó un régimen de "flotación fija" desde septiembre de 1977. Éste se ha convertido en un importante problema de política macroeconómica frente a las altas tasas de inflación actuales y los grandes ingresos proyectados por concepto de la venta de petróleo. Un mejor conocimiento del comportamiento posdevaluatorio de la demanda de moneda nacional, sería muy valioso para el establecimiento de una paridad flotante controlable. 


\section{BIBLIOGRAFIA}

Alexander, S. (1952), "The Effects of Devaluation on a Trade Balance", International Monetary Fund, Staff Papers, 2, pp. 263-78.

Banco de México (1945-1979), Informes Anuales, México, D. F.

(1978), "Producto Interno Bruto y Gasto: 1960-1977", Serie Información Económica, Subdirección de Investigación Económica y Bancaria, México, D. F.

(1979), "Bibliografía sobre Investigación Monetaria para México: 1965-1978", Serie Documentos de Investigación, 6, Subdirección de Investigación Económica y Bancaria, México, D. F.

Blejer, M. (1977), Dinero, precios y la balanza de pagos: la experiencia de México, 1960-1973, Centro de Estudios Monetarios Latinoamericanos, México, D. F.

Brothers, D. y Solís, L. (1966), Mexican Financial Development, University of Texas Press, Austin.

Bueno, G. (1971), "The Structure of Protection in Mexico", en Bela Balassa, ed., The Structure of Protection in Developing Countries, John Hopkins Press, Baltimore.

CEPAL-NAFINSA (1971), La Politica Industrial en el Desarrollo de México, México, D. F.

Cooper, R. (1971a), "Devaluation and Aggregate Demand in Aid Receiving Countries", en J. Bhagwati y al., Trade, Balance of Payments and Growth, North-Holland, Amsterdam.

(1971b), "Currency Devaluation in Developing Countries" Essays in International Finance, 86, International Finance Section, Princeton University, Princeton.

Diaz-Alejandro, C. (1963), "A Note on the Impact of Devaluation and the Redistributive Effect", Journal of Political Economy, pp. 577-80.

(1965), Currency Devaluation in a Semi-Industrialized Coum try, MIT Press, Cambridge.

Dornbusch, R. (1973a), "Currency Depreciation, Hoarding and Relative Prices", Journal of Political Economy, 81, pp. 893-915.

(1973b), "Devaluation, Money and Nontraded Goods", American Economic Review, 63, pp. 871-80.

(1976), "The Theory of Flexible Exchange Rate Regimes and Macroeconomic Policy", Scandinavian Journal of Economics, 78, pp. 255. 75.

Foley, D. y M. Sidrauski (1971), Monetary and Fiscal Policies in a Growing Economy, The MacMillan Company, London.

Foley, D. (1975), "On two Specifications of Assets Markets Equilibrium", Journal of Political Economy.

Gerakis, A. (1964), "Recession in the Initial Phase of a Stabilization Program: the Experience of Finland", IMF Staff Papers, 11, pp. 434-45.

Haberler, G. (1949), "The Market for Foreign Exchange and the Stability of the Balance of Payments", Kyklos, 3, pp. 193-218.

Hahn, F. (1959), "The Balance of Payments in a Monetary Economy", Review of Economic Studies, 26, pp. 110-25. 
Jorgenson, D. (1963), "Capital Theory and Investment Behavior", American Economic Review, pp. 247-59.

Kouri, P. y J. Braga de Macedo (1978), "Exchange Rates and the International Adjustment Process", Brookings Papers on Economic Activity, 1.

Krugman, P. y L. Taylor (1978), "The Contractionary Effects of Devaluation", Journal of International Economics.

Negishi, T. (1968), "Approaches to the Analysis of Devaluation", International Economic Review, 9, pp. 218-27.

Oficina de Asesores del C. Presidente de la República (1978), Encuesta sobre la actividad económica empresarial, México, D. F.

Ortiz, G. y L. Solís (1978), "Financial Structure and Exchange Rate Experience, México: 1954-1977", Journal of Development Economics (por publicarse).

Ortiz-Mena, A. (1969), "Desarrollo Estabilizador: una década de estrategia económica en México", El Mercado de Valores, 44, Nacional Financiera, S. A., México, D. F.

Reynolds, C. (1977), "Por qué el 'desarrollo estabilizador' de México fue en realidad desestabilizador", El Trimestre Económico, 64, pp. 997-1023.

Robinson, J. (1937), "The Foreign Exchanges", in Essays in the Theory of Unemployment, chap. 1, New York.

Solis, L. (1979), A Monetary Will-o'-the Wisp: Pursuit of Equity through Deficit Spending, por publicarse, Pergamon Press, New York.

Tobin, J. (1969), "A General Equilibrium Approach to Monetary Theory", Journal of Money, Credit and Banking, Febrero.

Zepeda, M. (1979), "Análisis gráfico del comportamiento de la economía", Oficina de Asesores del C. Presidente, mimeo., marzo, México, D. F. 